\title{
Racial Differences in Primary Care Opioid Risk Reduction Strategies
}

\author{
William C. Becker, $M D^{1}$ \\ Joanna L. Starrels, MD, MS \\ Moonseong Heo, $\mathrm{PbD}^{2}$ \\ Xuan Li, MS ${ }^{2}$ \\ Mark G. Weiner, $M D^{3}$ \\ Barbara J. Turner, MD, MSEd ${ }^{3}$ \\ 'Yale University School of Medicine, \\ New Haven, Connecticut \\ ${ }^{2}$ Albert Einstein College of Medicine \\ and Montefiore Medical Center, Bronx, \\ New York \\ ${ }^{3}$ University of Texas Health Science \\ Center and University Health System, \\ San Antonio, Texas
}

\begin{abstract}
PURPOSE Racial disparities in treating pain with opioids are widely reported; however, differences in use of recommended strategies to reduce the risk of opioid misuse by race/ethnicity have not been evaluated.

METHODS In a retrospective cohort of black and white patients with chronic noncancer pain prescribed opioid analgesics for at least 3 months, we assessed physicians' use of 3 opioid risk reduction strategies: (1) urine drug testing, (2) regular office visits (at least 1 visit per 6 months on opioids and within 30 days of an opioid change), and (3) restricted early opioid refills (receipt of a refill $>1$ week early less than twice). Nonlinear mixed effect regression models accounted for clustering within physician and adjusted additively for demographics, substance abuse, mental health and medical comorbidities, health care factors, and practice site.
\end{abstract}

RESULTS Of the 1,612 patients studied, $62.1 \%$ were black. Black patients were more likely than white patients to receive urine drug testing (10.4\% vs $4.1 \%)$, regular office visits (56.4\% vs $39.0 \%$ ), and restricted early refills $(79.4 \%$ vs $72.0 \%)$ $(P<.001$ for each). In fully adjusted models, black patients had significantly higher odds than their white counterparts of receiving regular office visits (odds ratio $=1.51 ; 95 \%$ confidence interval, $1.06-2.14$ ) and restricted early refills (odds ratio $=1.55 ; 95 \%$ confidence interval, $1.03-2.32$ ), but not urine drug testing (odds ratio $=1.41 ; 95 \%$ confidence interval, 0.78-2.54).

CONCLUSIONS In this cohort of primary care patients receiving opioid analgesics on a long-term basis, use of risk reduction strategies was very limited overall; however, black patients were more likely than white patients to receive 2 of 3 guideline-recommended strategies. These data raise questions about lax monitoring, especially for white patients taking opioids long term.

Ann Fam Med 2011;9:219-225. doi:10.1370/afm.1242.

\section{INTRODUCTION}

S tudies in varied health care settings including primary care practices report that physicians are less likely to prescribe opioid analgesics to treat pain in black compared with white patients, even after adjusting for severity of illness and other clinically relevant factors. ${ }^{1-4}$ This inequity in pain management is not based on evidence of greater opioid misuse among black patients; in fact, studies find that whites are more likely than blacks to misuse prescription opioids ${ }^{5,6}$ or that race is not a factor. ${ }^{7,8}$ Researchers hypothesize that differential opioid prescribing may reflect a lack of physician training and confidence in managing pain in minority groups as well as limited evidence-based protocols for managing pain with opioids, leading physicians to rely on stereotypes to guide decision making. ${ }^{9,10}$

Most chronic pain management occurs in primary care settings, where physicians must decide whether to prescribe opioids and, if these medications are prescribed, how to monitor patients for misuse. Given the increasing rates of opioid misuse and diversion, and their serious consequences including overdose and death, ${ }^{11-13}$ as well as physicians' inability 
to judge risk of opioid misuse accurately, ${ }_{1}^{14}$ it is important for all patients taking opioids to be monitored for safety. Recently, the American Academy of Pain Medicine and the American Pain Society ${ }^{15}$ and other experts ${ }^{16}$ have recommended that physicians should implement a structured treatment monitoring plan to reduce the risks associated with long-term opioid use. Few primary care physicians appear to have adopted recommended strategies, however. ${ }^{17-19}$

In light of reports of racial disparities in physician management of pain with more restrictive use of opioids for black compared with white patients, ${ }^{1-4}$ we hypothesized that racial differences exist in the opioid risk reduction strategies used for patients prescribed opioids long term for chronic pain. Specifically, we hypothesized that black patients would be more likely than white patients to receive urine drug testing, regular office visits, and restricted early refills.

\section{METHODS}

\section{Study Sample}

This retrospective study was conducted in 8 primary care practices affiliated with the University of Pennsylvania Health System in Philadelphia, Pennsylvania, from January 1, 2004, through July 31, 2008. The university's Institutional Review Board approved this project. All practices share an electronic health record containing data on demographics, diagnoses, laboratory tests, visit attendance, and prescribed medications. Patients aged 18 years or older were eligible if they met the following criteria: (1) had at least 3 practice visits demonstrating longitudinal care ${ }_{i}(2)$ had a recorded International Classification of Diseases, Ninth Revision, Clinical Modification (ICD-9-CM) diagnosis of musculoskeletal or neuropathic pain (ICD-9-CM codes $053.1 \mathrm{x}, 250.6 \mathrm{x}, 337.2 \mathrm{x}, 350.1 \mathrm{x}, 350.2 \mathrm{x}, 352.1 \mathrm{x}, 353 . \mathrm{xx}-$ 357.xx, 710.xx, 711.xx, 712.xx-716.xx, 719.xx-7.24.xx, 726.xx, 727.xx, 729.0x, 729.1x, 729.2x, 729.5x, 738.4x, $738.5 \mathrm{x}$ ); and (3) received long-term opioid treatment defined as 3 or more monthly prescriptions from a primary care physician for opioid analgesics (at least 21 days apart) within a 6-month period, similar to other studies. ${ }^{6,20}$ We excluded patients with a cancer diagnosis (140.xx-208.xx) except for nonmelanoma skin cancer or a remote history of prostate cancer as these are unlikely to require chronic pain management. We also excluded patients whose race was not black or white because they constituted less than $3 \%$ of the sample. We assigned a primary care physician to each patient based on whom the patient saw for a majority of visits during the study period; the monitoring occurring during the study period for the patient was attributed to that physician.

\section{Opioid Analgesic Treatment}

In study practices, all medications must be prescribed electronically. For this analysis, we included all prescriptions for oral, transdermal, or intranasal opioid analgesics but excluded intravenous or intramuscular opioid formulations and sublingual buprenorphine (which is not approved by the Food and Drug Administration for the treatment of pain). We also excluded the weak opioid tramadol, which has a lower risk of misuse. ${ }^{21}$ Other exclusions were absence of key data elements (ie, number of pills dispensed or directions on use), an indeterminate start date, or prescriptions rewritten within 3 days. The duration of long-term opioid treatment in this study commenced with the start date of the first opioid prescription and ended 30 days after the final prescription start date.

\section{Dependent or Outcome Variables}

The study outcomes were 3 opioid risk reduction strategies: (1) urine drug testing, (2) regular office visits, and (3) restricted early refills. Receipt of a urine drug test was defined as at least 1 completed urine drug test during the period of opioid treatment. Reflecting consensus panel recommendations, ${ }^{15,22}$ a regular office visit was defined as a primary care visit at least once within each 6-month interval that the patient received prescribed opioid analgesics and 30 days before or after an increase in an opioid dose or prescription of a new opioid. A restriction on early opioid refills was defined as existence of fewer than 2 occasions on which the patient received an opioid prescription more than 7 days before the previous prescription should have been completed if taken as directed. The duration of each opioid prescription was calculated from the number of pills dispensed divided by the number of pills to be taken per day according to directions. For prescriptions written "as needed" or "PRN," we conservatively assumed consumption was at the maximal prescribed dose, for example, 2 pills every 4 hours (or 12 per day) for prescriptions written "1-2 pills every 4-6 hours." All of the study practices had residents in training, but none had formal or structured educational interventions addressing opioid risk reduction during the study.

\section{Independent Variables}

The main independent variable was self-identified race, categorized as black or white. Demographic variables included age, sex, and median household income of the patient's zip code based on census data. ${ }^{23}$ Clinical variables were defined from coded diagnoses from inpatient or outpatient encounters. Because patients with co-occurring substance abuse are more likely to misuse prescribed opioids, ${ }^{7,8,24}$ we created a variable called problem substance use that was based on a diagnosis of 
abuse, dependence, poisoning, withdrawal, or substanceinduced psychosis during the study period for any of the following substances: alcohol, cocaine, amphetamines, cannabis, or benzodiazepines (ICD-9-CM codes available from authors). For opioids, problem substance use was based on codes before the first opioid prescription in the study period because we sought to isolate a history of opioid misuse as a risk factor for current misuse (ICD-9-CM codes also available). We also created a variable for tobacco use based on a diagnostic code of tobacco use disorder (ICD-9-CM 305.1).

Mental health disorders have also been associated with opioid misuse ${ }^{8,24}$; thus, we created a mental health variable based on a diagnosis of anxiety, depression, bipolar disorder, posttraumatic stress disorder, or schizophrenia (ICD-9-CM codes available). We identified medical comorbidities using a method adapted from that of Elixhauser and colleagues ${ }^{25}$ and included them as a categorical variable based on number of comorbidities (from none to 3 or more), as an estimate of overall severity of medical comorbidity. In regard to health care factors, we created a variable for the duration of long-term opioid treatment, as described. As an indicator of patient adherence to care, we created a variable for the proportion of scheduled primary care appointments attended within 2 years. We also created a categorical variable defining the intensity of primary care use with 3 levels: (1) higher if a patient had at least 1 visit in each 6 -month period on treatment and was in the highest quartile of monthly office visits; (2) lower if a patient had at least one 6 -month period without a visit and was in the lowest quartile of monthly office visits; and (3) intermediate for the remaining patients. This 3-level variable was used only in the analysis of urine drug testing and early opioid refills. Finally, to adjust for potential effects of practice site on risk reduction strategies, we included each patient's primary care practice site as a covariate.

\section{Statistical Analysis}

We conducted univariate analyses to examine associations of patient race and other covariates with the 3 outcome variables using $\chi^{2}$ tests for categorical variables and $t$ tests for continuous variables. To account for the hierarchical nature of the data with patients clustered within physician, our multivariate analysis used nonlinear mixed effect logistic regression models with physician included as a random effect. For each outcome variable, we built a nonlinear model that additively adjusted for patient demographics, substance abuse, mental health and medical comorbidities, and health care factors. Finally, practice site was added as a fixed effect. For analysis of urine drug testing, 2 of the practices, having a total of 274 patients, were removed from analysis because they performed only 1 or no urine drug tests among their study patients. We used SAS version 9.1 (SAS Institute Inc, Cary, North Carolina) to conduct all analyses.

\section{RESULTS}

The 8 primary care practices had a total of 4,057 patients who had 3 or more opioid prescriptions, of whom 1,612 (40\%) met our inclusion criteria (Figure 1). Most patients were excluded because they had not been prescribed opioids long term (ie, had $<3$ opioid prescriptions at least 21 days apart within 6 months).

In each practice, a median of 24 physicians (interquartile range, 6-47) treated at least 1 study patient. Among these physicians, the median number of study patients treated was 2 (interquartile range, 1-8) with a maximum of 163 . In the final study cohort, $8.0 \%$ of patients received urine drug testing, $49.8 \%$ had regular office visits, and $76.6 \%$ had restricted early refills.

Nearly two-thirds of the 1,612 study patients were black, and black patients were more likely to receive each opioid risk reduction strategy when compared with white patients (Table 1). Black patients were more likely than white patients to be female and to live in neighborhoods having a low-income zip code. In regard to factors associated with greater risk of misuse, black patients were more likely to have diagnosed problem substance use and tobacco use, but were not more likely to have a diagnosed mental health disorder. The pain-related conditions osteoarthritis and lumbago were more common in blacks than in whites. In addition, black patients had more medical comorbidities.

The mean duration of opioid treatment in the cohort was nearly 2 years and did not differ by race (Table 1). Blacks had more primary care visits but poorer adherence to scheduled appointments. The 2

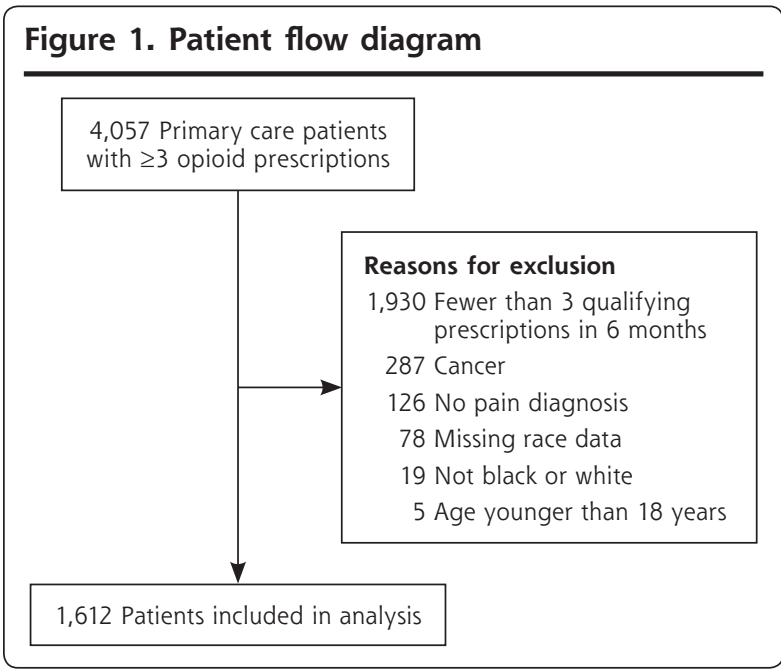


practices excluded from the urine drug testing models had significantly smaller proportions of black patients when compared with the remainder of the cohort (6.6\% vs $73.5 \% ; P<.001)$.

Table 2 displays the effect of additively adjusting for sets of patient, clinical, and health care variables on the odds of black patients receiving opioid risk reduction strategies when compared with their white counterparts. The far left column shows the unadjusted association of black race with each strategy. Subsequent columns moving to the right show the change in this association with sequential addition of each set of variables to the model. The Table 1. Patient Characteristics

\begin{tabular}{|c|c|c|c|c|}
\hline \multirow[b]{2}{*}{ Characteristic } & \multirow[b]{2}{*}{$\begin{array}{c}\text { All } \\
\text { Patients } \\
(\mathrm{N}=1,612)\end{array}$} & \multicolumn{3}{|c|}{ Racial Group } \\
\hline & & $\begin{array}{c}\text { Black } \\
\text { Patients } \\
(n=1,001)\end{array}$ & $\begin{array}{c}\text { White } \\
\text { Patients } \\
(n=611)\end{array}$ & $\begin{array}{c}P \\
\text { Value }^{a}\end{array}$ \\
\hline \multicolumn{5}{|l|}{ Outcome variables } \\
\hline Urine drug testing, any, No. (\%) & $129(8.0)$ & $104(10.4)$ & $25(4.1)$ & $<.001$ \\
\hline Regular office visits, No. (\%) & $803(49.8)$ & $565(56.4)$ & $238(39.0)$ & $<.001$ \\
\hline Restricted early refills, No. (\%) & $1,235(76.6)$ & $795(79.4)$ & $440(72.0)$ & $<.001$ \\
\hline \multicolumn{5}{|l|}{ Demographics } \\
\hline Age, mean (SD), years & $54.1(15.5)$ & $53.6(14.9)$ & $54.7(16.4)$ & .17 \\
\hline Women, No. (\%) & $1,070(66.4)$ & $708(70.7)$ & $362(59.3)$ & $<.001$ \\
\hline Household income, No. (\%) & & & & $<.001$ \\
\hline$<\$ 25,000$ & $407(25.3)$ & $380(38.0)$ & $27(4.4)$ & \\
\hline$\$ 25,000-\$ 34,999$ & $546(33.9)$ & $484(48.4)$ & $62(10.2)$ & \\
\hline$\$ 35,000-\$ 69,999$ & $544(33.8)$ & $129(12.9)$ & $415(67.9)$ & \\
\hline$\geq \$ 70,000$ & $115(7.1)$ & $8(0.8)$ & $107(17.5)$ & \\
\hline \multicolumn{5}{|l|}{ Substance abuse ${ }^{c}$} \\
\hline Problem substance use, No. (\%) & $174(10.8)$ & $130(13.0)$ & $44(7.2)$ & $<.001$ \\
\hline Tobacco use, No. (\%) & $259(16.1)$ & $187(18.7)$ & $72(11.8)$ & $<.001$ \\
\hline \multicolumn{5}{|l|}{ Mental and physical health } \\
\hline Mental health disorder, ${ }^{c}$ No. (\%) & $780(48.4)$ & $478(47.8)$ & $302(49.3)$ & .51 \\
\hline \multicolumn{5}{|l|}{ Pain diagnosis, ${ }^{\mathrm{d}}$ No. (\%) } \\
\hline Osteoarthritis & $693(43.0)$ & $482(48.2)$ & $211(34.5)$ & $<.001$ \\
\hline Rheumatoid arthritis & $130(8.1)$ & $83(8.3)$ & $47(7.7)$ & .66 \\
\hline Lumbago & $830(51.5)$ & $536(53.6)$ & $294(48.1)$ & .03 \\
\hline Cervical/thoracic spine pain & $390(24.2)$ & $234(23.4)$ & $156(25.5)$ & .33 \\
\hline Sciatica & $375(23.3)$ & $225(22.5)$ & $150(24.6)$ & .34 \\
\hline Neuropathic pain & $427(26.5)$ & $279(27.9)$ & $148(24.2)$ & .11 \\
\hline Fibromyalgia & $260(16.1)$ & $160(16.0)$ & $100(16.4)$ & .84 \\
\hline Medical comorbidities, ${ }^{c}$ No. (\%) & & & & $<.001$ \\
\hline None & $250(15.5)$ & $110(11.0)$ & $140(22.9)$ & \\
\hline 1 & $412(25.6)$ & $224(22.4)$ & $188(30.8)$ & \\
\hline 2 & $385(23.9)$ & $238(23.8)$ & $147(24.1)$ & \\
\hline$\geq 3$ & $565(35.1)$ & $429(42.9)$ & $136(22.3)$ & \\
\hline \multicolumn{5}{|l|}{ Health care factors } \\
\hline $\begin{array}{l}\text { Duration of long-term opioid } \\
\text { treatment, mean (SD), weeks }\end{array}$ & $100.0(62.9)$ & $101.9(64.4)$ & $96.8(60.4)$ & .11 \\
\hline $\begin{array}{l}\text { Appointment attendance rate, } \\
\text { median (SD) }\end{array}$ & $0.65(0.15)$ & $0.62(0.15)$ & $0.69(0.14)$ & $<.001$ \\
\hline Primary care use, ${ }^{e}$ No. (\%) & & & & .002 \\
\hline Lower & $256(15.9)$ & $135(13.5)$ & $121(19.8)$ & \\
\hline Intermediate & $1,008(62.5)$ & $634(63.3)$ & $374(61.2)$ & \\
\hline Higher & $348(21.6)$ & $232(23.2)$ & $116(19.0)$ & \\
\hline \multicolumn{5}{|c|}{$\begin{array}{l}{ }^{\text {a }} \chi^{2} \text { test for categorical variables; } t \text { test for continuous variables. } \\
\text { b Median annual household income in patient's ZIP code. } \\
\text { ' Based on physician International Classification of Diseases, Ninth Revision, Clinical Modification (ICD-9-CM) coding. } \\
\text { d Based on physician ICD-9-CM coding; patients may have multiple pain diagnoses. } \\
\text { e Lower = at least one } 6 \text {-month period without a visit and bottom quartile in office visit frequency; Higher = at } \\
\text { least one visit in each 6-month period and highest quartile in office visit frequency; Intermediate = remaining } \\
\text { patients. }\end{array}$} \\
\hline
\end{tabular}

far right column shows the odds ratios in the fully adjusted model.

In the unadjusted model, black patients were 1.63 times more likely to receive urine drug for clustering of patients within physicians, the odds ratio was no longer significant (Table 2). After adjustment for all sets of covariates, the odds of urine drug testing for blacks vs whites remained nonsignificant. The adjusted odds of regular office visits were 2.03-fold greater for black vs white patients and did not diminish with adjustment for clustering of patients within physician. The association of black race with regular office visits was progressively attenuated by adding patient, clinical, and health care factors, but the odds of having regular office visits were still $51 \%$ higher for black vs white patients in the fully adjusted model. Similarly, the unadjusted association of black race with restricted early refills was significant, with an odds ratio of 1.48 . In the fully adjusted model, black race remained significantly associated with 1.55-fold higher odds of restricted early refills.

\section{DISCUSSION}

In this large cohort of primary care patients treated for an average of 2 years with opioid analgesics for chronic noncancer pain, black patients were significantly more likely to receive recommended opioid risk reduction strategies than white patients. The adjusted odds of receiving regular office visits and restricted tests; however, after adjustment 
Table 2. Odds of Receipt of Opioid Risk Reduction Strategies for Black vs White Patients ${ }^{a}$

\begin{tabular}{|c|c|c|c|c|c|c|c|}
\hline \multirow[b]{2}{*}{ Strategy } & \multicolumn{7}{|c|}{ Additive Adjustment for Sets of Variables } \\
\hline & Unadjusted & Clustering $^{b}$ & Demographicsc & $\begin{array}{c}\text { Substance } \\
\text { Abuse }^{d}\end{array}$ & Comorbidities $^{e}$ & $\begin{array}{l}\text { Health Care } \\
\text { Factors }^{f}\end{array}$ & $\begin{array}{l}\text { Practice } \\
\text { Site }\end{array}$ \\
\hline Urine drug testing ${ }^{g}$ & $\begin{array}{c}1.63 \\
(1.03-2.59) \\
P=.04\end{array}$ & $\begin{array}{c}1.53 \\
(0.92-2.53) \\
P=.10\end{array}$ & $\begin{array}{c}1.44 \\
(0.82-2.54) \\
P=.21\end{array}$ & $\begin{array}{c}1.45 \\
(0.82-2.58) \\
P=.20\end{array}$ & $\begin{array}{c}1.54 \\
(0.86-2.73) \\
P=.15\end{array}$ & $\begin{array}{c}1.56 \\
(0.87-2.78) \\
P=.14\end{array}$ & $\begin{array}{c}1.41 \\
(0.78-2.54) \\
P=.26\end{array}$ \\
\hline Regular office visits & $\begin{array}{c}2.03 \\
(1.65-2.49) \\
P<.001\end{array}$ & $\begin{array}{c}2.22 \\
(1.71-2.87) \\
P<.001\end{array}$ & $\begin{array}{c}1.74 \\
(1.28-2.38) \\
P<.001\end{array}$ & $\begin{array}{c}1.74 \\
(1.28-2.38) \\
P<.001\end{array}$ & $\begin{array}{c}1.66 \\
(1.21-2.28) \\
P<.01\end{array}$ & $\begin{array}{c}1.55 \\
(1.10-2.19) \\
P=.01\end{array}$ & $\begin{array}{c}1.51 \\
(1.06-2.14) \\
P=.02\end{array}$ \\
\hline Restricted early refills & $\begin{array}{c}1.48 \\
(1.17-1.87) \\
P<.01\end{array}$ & $\begin{array}{c}1.60 \\
(1.22-2.10) \\
P<.01\end{array}$ & $\begin{array}{c}1.48 \\
(1.06-2.08) \\
P=.02\end{array}$ & $\begin{array}{c}1.50 \\
(1.07-2.10) \\
P=.02\end{array}$ & $\begin{array}{c}1.50 \\
(1.01-2.11) \\
P=.02\end{array}$ & $\begin{array}{c}1.56 \\
(1.06-2.31) \\
P=.03\end{array}$ & $\begin{array}{c}1.55 \\
(1.03-2.32) \\
P=.04\end{array}$ \\
\hline
\end{tabular}

Note: Values are odds ratios (95\% confidence intervals) and $P$ values.

a Nonlinear mixed effect regression models adjusting additively for sets of patient, clinical, and health care variables.

${ }^{b}$ Clustering of patients within physician.

' Includes sex, age, median household income of neighborhood.

${ }^{\mathrm{d}}$ Includes problem substance use (alcohol, nonopiates, and opioids), tobacco use.

${ }^{e}$ Mental health and medical comorbidities.

${ }^{\mathrm{f}}$ Includes duration of long-term opioid treatment, appointment attendance rate, and primary care use category. Primary care use category was not included in the regular office visits models.

${ }^{9}$ Urine drug testing analysis excludes 2 practices that performed only 1 test or no tests in patients ( $\mathrm{n}=274$ patients).

early opioid refills remained increased for black vs white patients after adjustment for diverse patient and health care factors. Approximately $10 \%$ of black patients received urine drug testing compared with only $4 \%$ of their white counterparts; after adjustment, the odds were still higher for black patients but no longer significantly so. Use of the other 2 risk reduction strategies was also low, with only one-half of the cohort receiving regular office visits and roughly $25 \%$ prescribed multiple early opioid refills. This poor performance of longterm opioid treatment monitoring supports widespread calls for improved quality of care to promote safe prescribing of these potentially dangerous medications. ${ }^{26}$

To our knowledge, this is the first study to examine racial differences in the use of risk reduction strategies for patients treated long term with opioids for chronic noncancer pain. Our novel findings extend those of several related studies. For example, Kunins and colleagues ${ }^{27}$ found pregnant black women were 1.5 times more likely to undergo urine screening for illicit drug use at prenatal visits than white women, but race was not associated with a positive test result. Our results also complement studies reporting that physicians underestimate the severity of pain in black patients ${ }^{28}$ and prescribe opioids for pain more commonly to white than to black patients. ${ }^{29-31}$ Taken together, these data indicate physicians are more cautious about initiating opioid therapy in black patients and, once the medication is started, monitor black patients more closely than whites.

On the other hand, our finding that black patients on chronic opioid therapy were seen in the office more regularly than white patients contrasts with findings of numerous studies reporting that black persons are less likely to use primary care services. ${ }^{32-34}$ Lower use of health care has been attributed in part to black patients' increased mistrust of clinicians and health care systems. ${ }^{35}$ We can only speculate as to why black patients were seen more regularly than white patients in our study. First, black patients had a greater number of medical comorbidities, which might have prompted more frequent monitoring visits. Second, black patients in our study population were more likely than white patients to have Medicaid insurance, so they might have had a lower or no copayment for visits. Copayments might have presented a greater barrier to frequent visits for white patients, although this seems unlikely given that white patients came from more affluent neighborhoods. Finally, the higher frequency of visits by black patients may reflect greater physician concern about the need to monitor for misuse or may reflect undertreatment of pain ${ }^{36}$ so that black patients had to return frequently to modify opioid medications.

Our findings suggest a "reverse disparity" ${ }^{1137}$ in the use of these opioid monitoring strategies, whereby traditionally vulnerable groups receive care more consistent with expert recommendations and guidelines. In fact, our results raise the concern that physicians are inappropriately lax in monitoring white patients, who received lower quality of care in regard to these monitoring outcomes. This laxity contradicts evidence that the risk of prescription drug abuse is greater in whites than in other racial/ethnic groups. ${ }^{5,6}$

In our cohort, the association of race with urine drug testing was attenuated after adjustment for clustering within physician, suggesting some physicians may have contributed to this disparity more than others. This was the only outcome, however, for which accounting for clustering of patients within physicians 
diminished the association of race with the outcome. The monitoring practices of the clinician who had 163 patients on opioids long term were similar to the study averages and displayed no difference in monitoring between black and white patients; thus, excluding this high-volume prescriber's patients would not have changed our conclusions. Future studies should examine the role of variation in the monitoring practices of individual clinicians in contributing to these inequitable practices in monitoring persons on opioid analgesics.

Although we could not investigate the underlying causes for the across-the-board low rates of implementation of risk reduction strategies, lack of physician training, time, and other practice resources are likely to be major barriers. Another factor may be the dearth of evidence demonstrating the effectiveness of risk reduction strategies in improving misuse outcomes. ${ }^{38} \mathrm{With}$ emerging evidence of an increased overdose risk with higher opioid dosages, ${ }^{39}$ development of safety-driven treatment and monitoring algorithms becomes even more imperative. Systems-based, safety-enhancing approaches, common in the use of coumadin ${ }^{40}$ and insulin, ${ }^{41}$ and emerging with opioids, ${ }^{42}$ are needed to improve the overall use of risk reduction strategies and reduce racial differences in monitoring. Indeed, several centers are pilot testing novel approaches such as use of automated monitoring reminders in the medical record ${ }^{43}$ medication monitoring programs staffed by midlevel clinicians within primary care clinics, ${ }^{42}$ and multidisciplinary, disease management clinics where monitoring protocols are standardized. ${ }^{44}$

We acknowledge limitations to our study. First, in this observational study, our findings cannot demonstrate causality; however, we were able to control for potential confounding variables, most notably diagnosed substance abuse and mental health disorders, which are associated with an increased risk of misuse. We relied on coded diagnoses to identify comorbidities including substance use disorders, and we may have missed clinical conditions or behaviors that would increase the need to monitor for misuse, or other unmeasured confounders. It is possible that this misclassification was differential, that is, that clinicians were more likely to code substance use disorder for black than white patients, which would bias results toward the null and attenuate the difference in models adjusting for substance use disorders. Second, despite our relatively large cohort, the low prevalence of urine drug testing limited our power to find a significant association. Furthermore, we removed 2 practices from urine drug testing models because they had little or no urine testing, these practices had predominantly white patients, so excluding them may have led to an underestimation of race's association with urine drug testing. Third, our cohort came from practices in a single, urban, university-affiliated health system, and the findings may therefore not be generalizable to other settings. Fourth, we did not evaluate the dose of opioids prescribed, however, standard of care is that all patients on long-term opioid therapy need to be monitored, regardless of dose. Last, we examined outcomes supported by consensus guidelines and assessable using the electronic health record. We could not ascertain other monitoring practices, such as use of treatment agreements, querying prescription-monitoring databases, and performing pill counts.

Many experts in the field of pain management advocate for a "universal precautions" approach to risk reduction for patients prescribed opioids for chronic noncancer pain. ${ }^{45}$ Our findings show that real-world clinical practice falls far short of this ideal. This evidence of racial differences in the management chronic noncancer pain should stimulate clinical and educational initiatives to ensure all patients are appropriately monitored. Further, physicians should recognize that patient race should not affect their use of their monitoring strategies. Outcomes research is also necessary to determine whether systems-based approaches with standardized monitoring strategies ameliorate these differences and improve overall monitoring rates.

To read or post commentaries in response to this article, see it online at http://www.annfammed.org/cgi/content/full/9/3/219.

Key words: Opioid analgesics; safety monitoring; disparities; urine drug testing; race/ethnicity; practice-based research; primary care

Submitted April 15, 2010; submitted, revised, November 18, 2010; accepted December 6, 2010.

Presented at the Society of General Internal Medicine 32nd Annual Meeting, Miami Beach, Florida, May 16, 2009.

Funding support: This work was supported through the Program of Research Integrating Substance Use Issues into Mainstream Health Care (PRISM), funded by the Robert Wood Johnson Foundation, the National Institute on Drug Abuse (NIDA), and the Substance Abuse and Mental Health Services Administration (SAMHSA).

Acknowledgments: The authors would like to thank Evelyn Crowley and Tom Ten Have.

\section{References}

1. Chen I, Kurz J, Pasanen M, et al. Racial differences in opioid use for chronic nonmalignant pain. J Gen Intern Med. 2005;20(7):593-598.

2. Olsen Y, Daumit GL, Ford DE. Opioid prescriptions by U.S. primary care physicians from 1992 to 2001. J Pain. 2006;7(4):225-235.

3. Pletcher MJ, Kertesz SG, Kohn MA, Gonzales R. Trends in opioid prescribing by race/ethnicity for patients seeking care in US emergency departments. JAMA. 2008;299(1):70-78.

4. Tamayo-Sarver JH, Dawson NV, Hinze SW, et al. The effect of race/ ethnicity and desirable social characteristics on physicians' decisions to prescribe opioid analgesics. Acad Emerg Med. 2003;10(11):1239-1248. 
5. Becker WC, Sullivan LE, Tetrault JM, Desai RA, Fiellin DA. Nonmedical use, abuse and dependence on prescription opioids among U.S. adults: psychiatric, medical and substance use correlates. Drug Alcohol Depend. 2008;94(1-3):38-47.

6. Edlund MJ, Steffick D, Hudson T, Harris KM, Sullivan M. Risk factors for clinically recognized opioid abuse and dependence among veterans using opioids for chronic non-cancer pain. Pain. 2007; 129(3):355-362.

7. Ives TJ, Chelminski PR, Hammett-Stabler CA, et al. Predictors of opioid misuse in patients with chronic pain: a prospective cohort study. BMC Health Serv Res. 2006;6:46.

8. Fleming MF, Balousek SL, Klessig CL, Mundt MP, Brown DD. Substance use disorders in a primary care sample receiving daily opioid therapy. J Pain. 2007;8(7):573-582.

9. Burgess DJ, van Ryn M, Crowley-Matoka M, Malat J. Understanding the provider contribution to race/ethnicity disparities in pain treatment: insights from dual process models of stereotyping. Pain Med. 2006;7(2):119-134.

10. Balsa Al, McGuire TG. Prejudice, clinical uncertainty and stereotyping as sources of health disparities. J Health Econ. 2003;22(1):89-116.

11. Kuehn BM. Efforts aim to curb opioid deaths, injuries. JAMA. 2009; 301(12):1213-1215.

12. Hall AJ, Logan JE, Toblin RL, et al. Patterns of abuse among unintentional pharmaceutical overdose fatalities. JAMA. 2008;300(22): 2613-2620.

13. Substance Abuse and Mental Health Services Administration, Office of Applied Studies. Drug Abuse Warning Network, 2007: National Estimates of Drug-Related Emergency Department Visits. Rockville, MD: Substance Abuse and Mental Health Services Administration; 2010. https://dawninfo.samhsa.gov/files/ED2007/DAWN2k7ED.pdf. Accessed January 12, 2010.

14. Katz NP, Sherburne S, Beach M, et al. Behavioral monitoring and urine toxicology testing in patients receiving long-term opioid therapy. Anesth Analg. 2003;97(4):1097-1102.

15. Chou R, Fanciullo GJ, Fine PG, et al; American Pain Society-American Academy of Pain Medicine Opioids Guidelines Panel. Clinical guidelines for the use of chronic opioid therapy in chronic noncancer pain. J Pain. 2009;10(2):113-130.

16. McLellan AT, Turner BJ. Chronic noncancer pain management and opioid overdose: time to change prescribing practices. Ann Intern Med. 2010;152(2):123-124.

17. Yanni LM, Weaver MF, Johnson BA, Morgan LA, Harrington SE, Ketchum JM. Management of chronic nonmalignant pain: a needs assessment in an internal medicine resident continuity clinic. J Opioid Manag. 2008;4(4):201-211.

18. Adams NJ, Plane MB, Fleming MF, Mundt MP, Saunders LA, Stauffacher EA. Opioids and the treatment of chronic pain in a primary care sample. J Pain Symptom Manage. 2001;22(3):791-796.

19. Bhamb B, Brown D, Hariharan J, Anderson J, Balousek S, Fleming MF. Survey of select practice behaviors by primary care physicians on the use of opioids for chronic pain. Curr Med Res Opin. 2006; 22(9):1859-1865.

20. Simon GE, VonKorff M, Barlow W, Pabiniak C, Wagner E. Predictors of chronic benzodiazepine use in a health maintenance organization sample. J Clin Epidemiol. 1996;49(9):1067-1073.

21. Cicero TJ, Inciardi JA, Muñoz A. Trends in abuse of Oxycontin and other opioid analgesics in the United States: 2002-2004. J Pain. 2005;6(10):662-672.

22. Fine PG, Portenoy RK; Ad Hoc Expert Panel on Evidence Review and Guidelines for Opioid Rotation. Establishing "best practices" for opioid rotation: conclusions of an expert panel. J Pain Symptom Manage. 2009;38(3):418-425.

23. US Census Bureau. Census 2000, Summary File 3.

24. Akbik H, Butler SF, Budman SH, Fernandez K, Katz NP, Jamison RN. Validation and clinical application of the Screener and Opioid Assessment for Patients with Pain (SOAPP). J Pain Symptom Manage. 2006;32(3):287-293.
25. Elixhauser A, Steiner C, Harris DR, Coffey RM. Comorbidity measures for use with administrative data [see comment]. Med Care. 1998;36(1):8-27.

26. McLellan AT, Turner B. Prescription opioids, overdose deaths, and physician responsibility. JAMA. 2008;300(22):2672-2673.

27. Kunins HV, Bellin E, Chazotte C, Du E, Arnsten JH. The effect of race on provider decisions to test for illicit drug use in the peripartum setting. J Womens Health (Larchmt). 2007;16(2):245-255.

28. Staton LJ, Panda M, Chen I, et al. When race matters: disagreement in pain perception between patients and their physicians in primary care. J Natl Med Assoc. 2007;99(5):532-538.

29. Todd KH, Samaroo N, Hoffman JR. Ethnicity as a risk factor for inadequate emergency department analgesia. JAMA. 1993;269(12): 1537-1539.

30. Singer AJ, Thode HC Jr. National analgesia prescribing patterns in emergency department patients with burns. J Burn Care Rehabil. 2002;23(6):361-365.

31. Cleeland CS, Gonin R, Baez L, Loehrer P, Pandya KJ. Pain and treatment of pain in minority patients with cancer. The Eastern Cooperative Oncology Group Minority Outpatient Pain Study. Ann Intern Med. 1997;127(9):813-816.

32. Basu J. Access to primary care: the role of race and income. J Health Soc Policy. 2001;13(4):57-73.

33. Ferrer RL. Pursuing equity: contact with primary care and specialist clinicians by demographics, insurance, and health status. Ann Fam Med. 2007;5(6):492-502.

34. Basu J, Clancy C. Racial disparity, primary care, and specialty referral. Health Serv Res. 2001;36(6 Pt 2):64-77.

35. Armstrong K, McMurphy S, Dean LT, et al. Differences in the patterns of health care system distrust between blacks and whites. J Gen Intern Med. 2008;23(6):827-833.

36. Green CR, Anderson KO, Baker TA, et al. The unequal burden of pain: confronting racial and ethnic disparities in pain. Pain Med. 2003;4(3):277-294.

37. Gurmankin AD, Polsky D, Volpp KG. Accounting for apparent "reverse" racial disparities in Department of Veterans Affairs (VA)based medical care: influence of out-of-VA care. Am J Public Health 2004;94(12):2076-2078.

38. Starrels JL, Becker WC, Alford DP, Kapoor A, Williams AR, Turner BJ. Systematic review: treatment agreements and urine drug testing to reduce opioid misuse in patients with chronic pain. Ann Intern Med. 2010;152(11):712-720.

39. Dunn KM, Saunders KW, Rutter CM, et al. Opioid prescriptions for chronic pain and overdose: a cohort study. Ann Intern Med. 2010; 152(2):85-92.

40. Chiquette E, Amato MG, Bussey HI. Comparison of an anticoagulation clinic with usual medical care: anticoagulation control, patient outcomes, and health care costs. Arch Intern Med. 1998;158(15):1641-1647.

41. Pieber TR, Brunner GA, Schnedl WJ, Schattenberg S, Kaufmann P, Krejs GJ. Evaluation of a structured outpatient group education program for intensive insulin therapy. Diabetes Care. 1995;18(5):625-630.

42. Wiedemer NL, Harden PS, Arndt IO, Gallagher RM. The opioid renewal clinic: a primary care, managed approach to opioid therapy in chronic pain patients at risk for substance abuse. Pain Med. 2007;8(7):573-584.

43. Trafton J, Martins S, Michel M, et al. Evaluation of the acceptability and usability of a decision support system to encourage safe and effective use of opioid therapy for chronic, noncancer pain by primary care providers. Pain Med. 2010;11(4):575-585.

44. Chelminski PR, Ives TJ, Felix KM, et al. A primary care, multi-disciplinary disease management program for opioid-treated patients with chronic non-cancer pain and a high burden of psychiatric comorbidity. BMC Health Serv Res. 2005;5(1):3.

45. Gourlay D, Heit H. Universal precautions: a matter of mutual trust and responsibility [comment]. Pain Med. 2006;7(2):210-211; author reply 212 